\title{
The OpenRadiation project: monitoring radioactivity in the environment by and for the citizens
}

\author{
J.F. Bottollier-Depois ${ }^{1, *}$, E. Allain ${ }^{2}$, G. Baumont ${ }^{2}$, N. Berthelot ${ }^{3}$, G. Darley ${ }^{1}$, F. Ecrabet ${ }^{1}$, T. Jolivet $^{3}$, \\ A. Lebeau-Livé ${ }^{1}$, V. Lejeune ${ }^{1}$, F. Quéinnec ${ }^{1}$, C. Simon ${ }^{4}$ and F. Trompier ${ }^{1}$ \\ ${ }^{1}$ IRSN, Institut de Radioprotection et de Sûreté Nucléaire, 92262 Fontenay-aux-Roses, France. \\ 2 IFFoRME, Institut Français des Formateurs Risques Majeurs et protection de 1'Environnement, 75010 Paris, France. \\ 3 Planète Sciences, 91130 Ris-Orangis, France. \\ ${ }^{4}$ Fablab de Sorbonne Université, 75005 Paris, France.
}

Received: 22 October 2019 / Accepted: 30 October 2019

\begin{abstract}
After the Fukushima accident, initiatives emerged offering the public the possibility to realise measurements of the radioactivity in the environment with various devices and to share data and experiences through collaborative tools. The objective of the OpenRadiation project is to offer the public the opportunity to perform measurements of the radioactivity using connected dosimeters on smartphones. The challenge is to operate such a system on a sustainable basis in normal situations and in order to be useful in an emergency situation. In normal situations, this project is based on a collaborative approach including pedagogical activities. In case of emergency situation, data from the field will be available in "real time" providing an opportunity for the emergency management and the communication with the public. The practical objectives are to develop i) a website centralising measurements using various dosimeters, providing dose rate maps with raw and filtered data and offering dedicated areas for specific projects and exchanges about data and ii) a dosimetric app using a connected dosimeter. This project is conducted within a partnership between organisms' representative of the scientific community and associations to create links with the public.
\end{abstract}

Keywords: OpenRadiation / citizen science / radioactivity measurement / environment

\section{Introduction}

As smartphones have developed into geolocated multi-task assistants, a plethora of applications related to healthcare and the environment has emerged, reflecting deep-felt concerns across society. These tend to empower both the individual and the community with which he or she wishes to share their results to become personally involved in taking action on these issues. They are also very easy to use and make monitoring more visible.

Measuring environmental quality is a major focus of interest (air quality outdoors, indoors, in different environments, etc.) and websites and applications have been developed to make the data collected by public bodies available. In particular, there are apps designed to go much further when combined with measurement devices, allowing a person looking up information to share data as well. This is what happened after the accident at the Fukushima nuclear power plant, when a whole generation of apps for use by the public to take geolocated radioactivity measurements in the

\footnotetext{
${ }^{*}$ Corresponding author: jeanfrancois.bottollier@irsn.fr
}

field sprung up spontaneously. What this reveals is a desire on the part of ordinary people to be able to assess the risks to which they are exposed themselves, in addition to knowing the measurements and estimates made by official and nongovernmental organisations (Lochard et al., 2019).

Given the increasing popularity of this new participatory science, the French Institute for Radiological Protection and Nuclear Safety (IRSN), the French Institute for trainers in major risks and environmental protection (IFFO-RME), Planète Sciences and the Sorbonne Universities' FabLab have set up a website to collect dose rate measurements taken by citizens in the environment, and have also developed a dose rate measurement device that can be connected to a smartphone. This is the OpenRadiation project described in this article.

\section{Project aims and challenges}

OpenRadiation is a project designed for individuals to measure ambient radioactivity, including radiation of terrestrial and cosmic origins as well as that generated by human activities. It is an open data and open source citizen science 
project. The OpenRadiation project has been designed to meet various objectives, in normal situations and in emergency situations.

In normal situations, OpenRadiation is a citizen science project that aims to:

- enable the public to measure radioactivity themselves as part of a collaborative initiative;

- integrate this system as part of an educational initiative targeted at specific groups, such as sixth formers and university students, local information committees around nuclear facilities, groups formed to address a specific project, etc.;

- add to existing data, especially with regard to mapping background radiation in the environment;

- be involved in radiological monitoring with a view to detecting abnormal events. This implies a need to set up an organisation for managing such events.

In an emergency situation, OpenRadiation is a platform that will be useful in managing the emergency response, with a view to:

- gathering together measurements taken by the public in the field and in real time;

- forward planning for mass data uploads and processing, and then for various stakeholders to share and use the data;

- enabling the public to be involved and provide data that could be used in assessing a radiological accident.

For the citizen, it is a chance to take part in a participatory science project, taking measurements that will be made available to the general public, the scientific community and to all the stakeholders involved in emergency response management, as well as being able to use the system to assess the risk they face themselves, especially in an emergency situation.

The challenge is to attract citizens in such a participatory science project on a sustainable basis under normal circumstances and in a radiological emergency situation.

Under normal circumstances, the following actions are needed to address this challenge:

- set up a sustainable system by developing communities that include different categories of people, such as schoolchildren, students, committees focusing on specific sites of interest, and people who are interested in contributing to a science project;

- obtain data that is robust enough to be used in science projects, and add to existing environmental measurement data compiled by institutions or NGOs, etc.;

- improve measurement quality and improve knowledge of the systems used (recommendations on taking measurements, improving calibration for connected sensors, etc.);

- manage abnormal measurements that are higher than background radiation: identifying true positives ("natural" hot spots that are not representative of the environment and that could trigger an alert) and false positives (malfunction, deliberately false value);

- manage a citizen science website: set up and coordinate the user community, moderate and maintain the website, etc.

In a radiological emergency situation, it is likely that masses of data will be uploaded by the public and the challenge then would be quite different:
- share measurements that are as reliable as possible so that they can be used to manage the emergency (decision making in the early stages, and for mid- and long-term post-accident management) and to assess the reliability of such measurements;

- communicate with the public: with the option to discuss what is happening with the user community, and especially with people who are on-site;

- manage a website in an emergency situation: the ability to absorb a huge amount of data, and deal with more intensive moderation/coordination tasks, etc.

\section{The OpenRadiation project}

OpenRadiation is made up of a website (https://www. openradiation.org) and a dose measurement app for use on a smartphone (https://apps.apple.com/us/app/openradiation/ id1246249831, https://play.google.com/store/apps/detailsid= org.openradiation).

The main functions of the website are for:

- collecting measurements that may have been taken using different types of sensor, received via the OpenRadiation app or directly on the website, and that may have been taken either at ground level or on a plane;

- mapping measurements, by default using raw data or using filters: dose range, time window, specific tag, etc.;

- discussing any questionable measurements: special functions have been developed for comments or for giving an opinion if needed;

- providing information for educational purposes: on the effects of ionising radiation, good practice relative to taking measurements, which dosimeter to choose, etc.;

- providing a dedicated space for facilitating interaction and data sharing for groups of citizens who wish to carry out specific "missions" in line with the project.

A dosimetry application for smartphones, combined with an external dosimeter, has been developed for taking measurements and sending them to the OpenRadiation database. The dosimetric quantity measured is the ambient dose equivalent rate given in $\mu \mathrm{Sv}$ per hour. Every measurement is accompanied by around fifty metadata used to contextualise data and designed for data analysis. Some of these metadata are automatically generated by the app (geolocation, start/end of measurement, type of detector, type of telephone, etc.) while others are entered by the user (indoors/outdoors measurement, on the ground/on a plane, weather conditions, etc.). Different types of dosimeter can be used with the app: a kit version to be assembled yourself, which has been developed for the project, or certain off-theshelf models that have been tested and recommended to interface with the app. In addition, five models, based on Muller Geiger counters or photodiodes, can be used with the OpenRadiation app and are proposed so far on the OpenRadiation website: Atom Tag (Geiger Muller (GM) tube, Bluetooth), Pocket Geiger type 6 (silicon diode, USB), Polismart 2 (GM tube, Bluetooth), Rium GM (GM tube, USB and Bluetooth early 2020) and Safecast (GM pancake, 


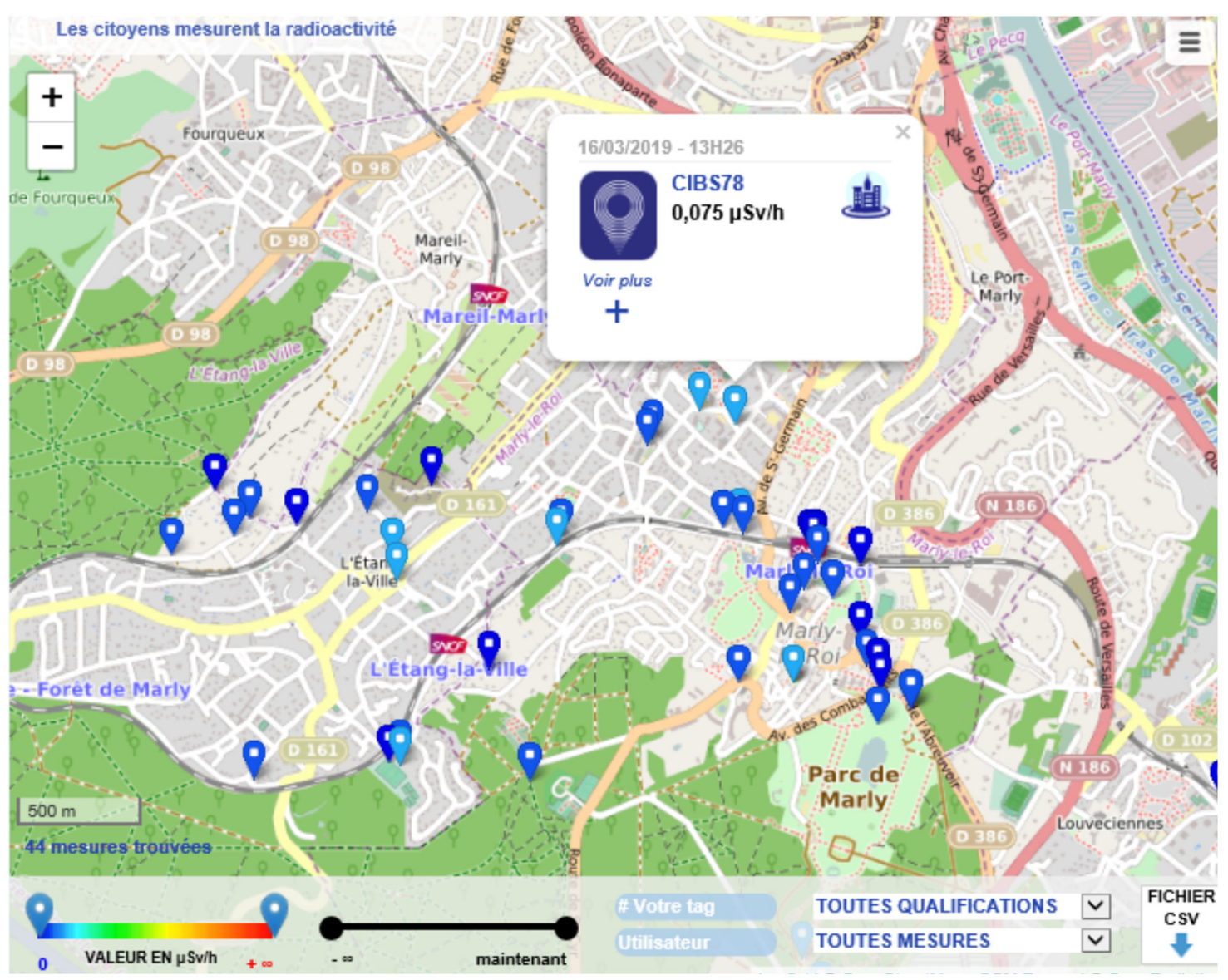

Fig. 1. Measurements realised by high school students from Louis de Broglie secondary school, Marly-le-Roi (France).

Bluetooth). It is also possible to submit measurement results taken using any kind of dose rate meters thanks to "manual" submission mode; the measurement will then be geolocated by the app at the time the data is recorded. These dosimeters have been characterised at reference facilities and compared to professional dosimeters under real use conditions. Calibration functions have been improved where deemed necessary. The app works in two modes, one for taking measurements on the ground and the other for taking measurements on a plane. Each is assigned a specific calibration function adapted to the different types of radiation encountered according to whether it is of terrestrial and/or cosmic origin. The app gives the user the option of taking a single measurement (manual start/end) or a series of measurements specifying the duration or the number of counts for each measurement and the total cycle time.

\section{Preliminary results}

The OpenRadiation website was launched for public use in October 2017. Since then, the dose measurement app has been upgraded twice, first in December 2018, to provide an interface for connecting different types of dosimeter available to buy online and the option to take a series of measurements and, second, in June 2019, to provide a special function for measuring cosmic radiation on a plane.

\subsection{First measurement statistics}

Since the website was launched, over 115000 measurements have been taken worldwide by around 90 users. Some of these measurements (35000) were taken using the OpenRadiation app, and the rest were taken using the Safecast dosimeter (Brown et al., 2016) which can be used to take a series of multiple measurements, uploading the data files directly to the OpenRadiation database. Most of the measurements taken with the Safecast dosimeter were made in the months following the launch of the system, since only the kit dosimeter and manual mode were available on the app in the first year, which limited the amount of measurements taken.

Figure 1 is an example of measurements performed by high school students from Louis de Broglie secondary school in Marly-le-Roi (France) and shows that values are coherent with the natural background in this area. In general, as shown in Figure 2, measurements have mostly been taken outdoors at ground level $(55 \%)$, aboard a plane (25\%) and on a vehicle on the road $(17 \%)$. The remaining $3 \%$ were taken indoors in the home or in town. Measurements were taken using all the different dosimeters recommended on the OpenRadiation website. Their number using the OpenRadiation app per type of dosimeter is given in Figure 3. Another interesting point is how have progressed the monthly measurements since the beginning. Indeed a global increase of the activity is observed 


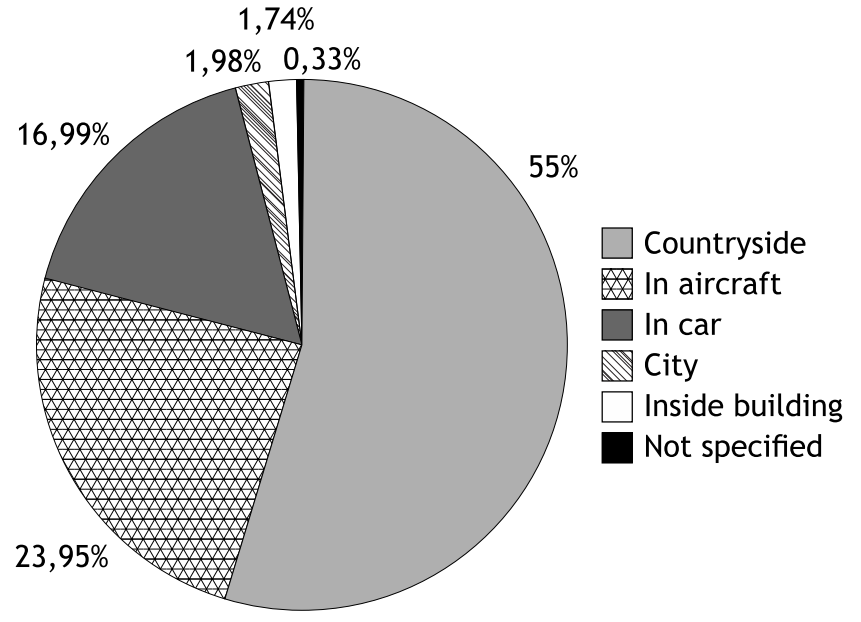

Fig. 2. Breakdown of measurement conditions using the OpenRadiation app.

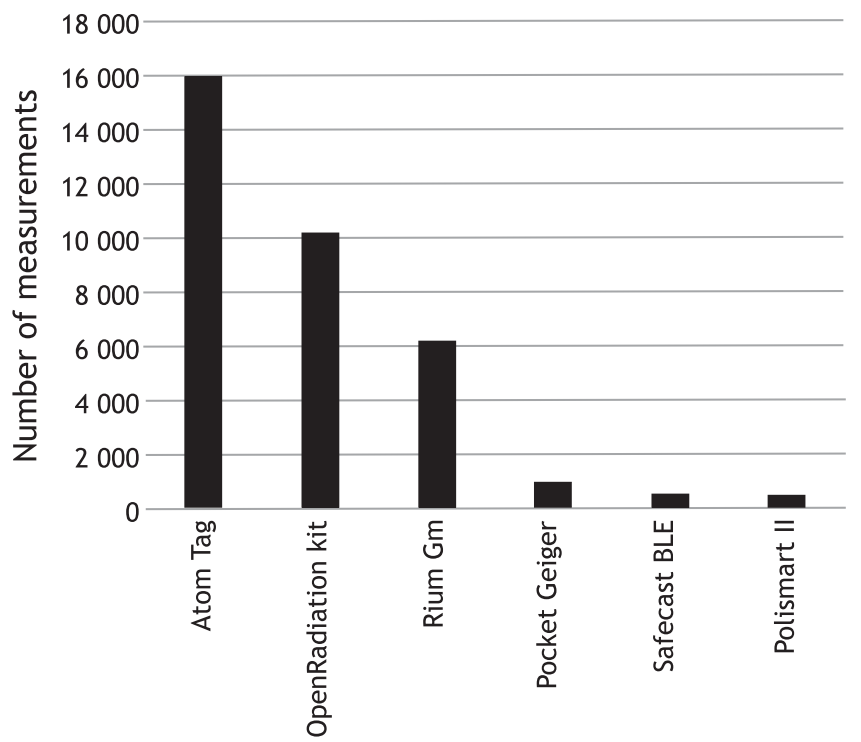

Fig. 3. Number of measurements taken using the OpenRadiation app per type of dosimeter.

from early 2019 , corresponding to the starting of the version with the option to take a series of measurements (Fig. 4).

The distribution of values measured outdoors at ground level is in line with the background radiation values expected in the environment (Fig. 5). The average measurement value is $110 \mathrm{nSv} / \mathrm{h}$. These measurements need to be analysed in more depth to examine the effect of variability between sensors and to evaluate any bias that could be due to higher measurements taken by people seeking a "hot spot" in the environment. For the sake of comparison, the values logged in mainland France by the RNM network (https://www.mesure-radioactivite.fr) range from $60 \mathrm{nSv} / \mathrm{h}$ at sea level to $120 \mathrm{nSv} / \mathrm{h}$ in the Massif Central (IRSN, 2016). Measurements taken on planes since June 2019 , with a specific calibration factor, agree satisfactorily with those taken using other types of dosimeter, including some so-called "reference" dosimeters, and with calculations

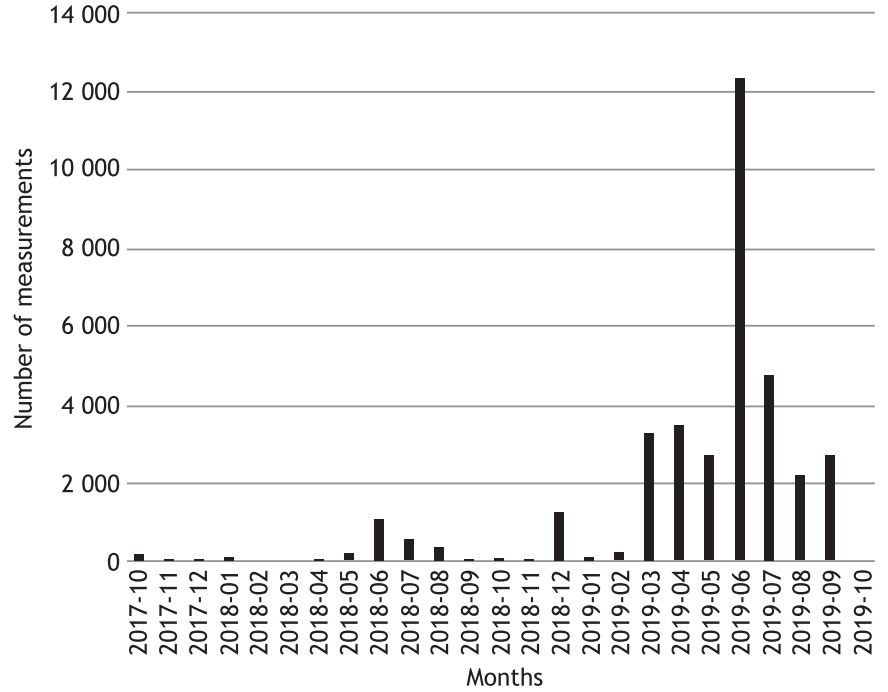

Fig. 4. Monthly measurement rate using the OpenRadiation app.

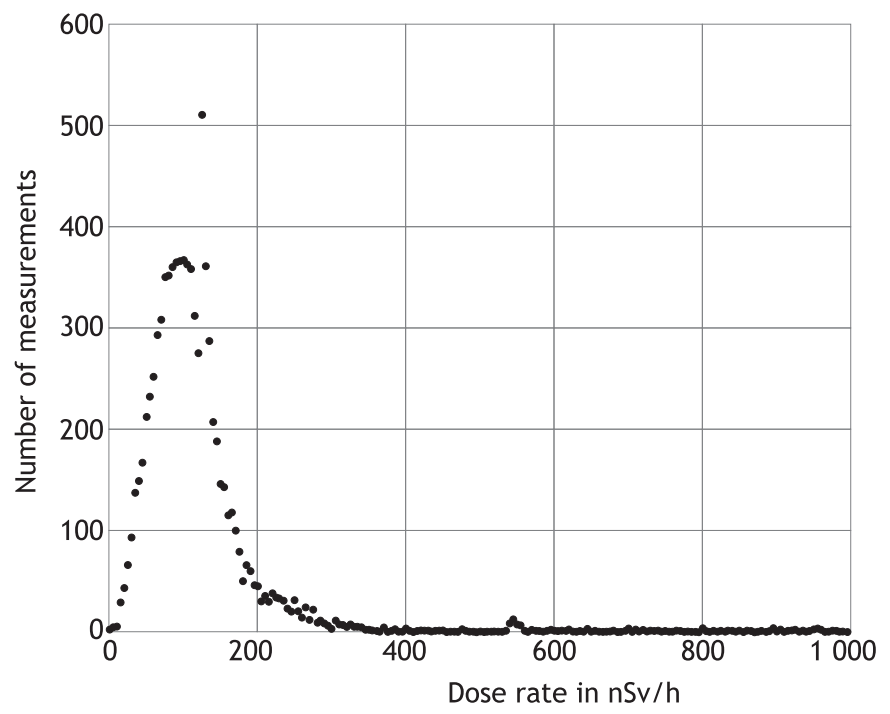

Fig. 5. Distribution of dose rate measurements taken outdoors on the ground at a height of 1 metre using the OpenRadiation app.

made using SIEVERTPN (Bottollier-Depois et al., 2007, https://www.sievert-system.org), the dose measurement tool used for air crew in France. Figure 6 compares measurements, aboard an aircraft during a flight between Paris and Shanghai on June 15th 2019, taken with an OpenRadiation dosimeter kit and a Tissue Equivalent Proportional Counter (TEPC) considered as the reference instrumentation for cosmic radiation dosimetry.

To ensure complete transparency, all raw data (no prior filtering) is available in real time as open data both to the public and to the scientific community (https://request.openradiation. net/download.html). The more such data there is, and the more reliable it is, the more it will be used. A number of practical applications making use of this data are being developed, for example for relaying alerts in the event that abnormally high values are detected, for the purposes of assessment in addition 


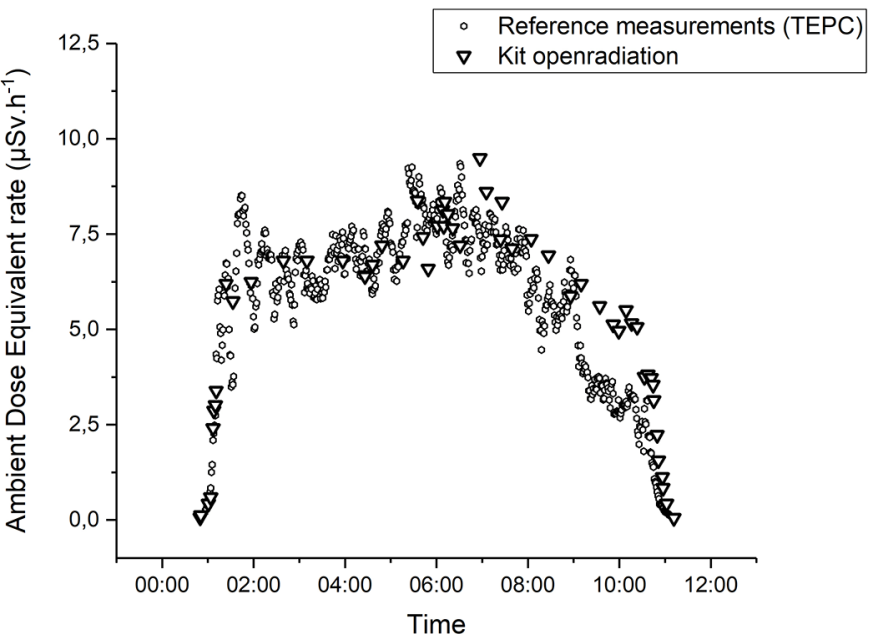

Fig. 6. In flight comparison of ambient dose equivalent rate measured with a Tissue Equivalent Proportional Counter (TEPC), considered as the reference instrumentation for cosmic radiation dosimetry, and an OpenRadiation detector (kit version with a SBM20 Geiger Muller tube). A specific calibration factor for cosmic radiation is used for the GM tube detector $\left(0.016 \mu \mathrm{Sv} \cdot \mathrm{h}^{-1} . \mathrm{cts}^{-1} . \mathrm{min}\right)$. Measurements were taken aboard an Airbus A380 during a flight between Paris and Shanghai on June 15th 2019.

to existing ambient measurements, for use in emergency response management in the event of a radiological accident, for collecting cosmic radiation measurements aboard planes in order to validate dosimetry models, etc.

\subsection{An emerging community}

A very broad range of people contribute to the database. They include individual users who have different reasons for doing so, for instance, "opportunists" (travel, holidays, work trip, etc.), or people who are curious or have a particular interest in measuring radioactivity (to find a "hot spot", or to learn more about their environment, etc.), and groups of users taking part in projects that have a more structured approach (defining an objective, a mission, etc.). The majority of measurements are taken by a small number of regular users, while many contributors only take a couple of measurements. It should be mentioned that, to date, no deliberately false values have been logged, even though there is no filtering system in place.

A study using OpenRadiation has, for example, been conducted as part of TERRITORIES (www.territories.eu), a European research project that takes a citizen science approach. This study assessed the radiological quality of a territory contaminated due to the accident at Chernobyl, at the scale of a village, getting pupils at the village school to take measurements themselves and analyse them with the help of scientists involved in the project (Bertho et al., 2019). Among other things, this study demonstrated that the pupils were really keen to be involved in the project, which in turn served to get the local population interested in the practical aspects of radiological protection (Lochard et al., 2019). This study also questioned the role of the expert in the context of a study that took a participatory approach to science, as highlighted in a recent publication (Schneider et al., 2019).

A number of different projects have been conducted by high school and university students, defining their own objectives with their teachers and helped by experts in some cases. For example, high school students at Albert Londres secondary school in Vichy compared measurements taken using the OpenRadiation dosimeter with those taken using Safecast. Students at IUT Paris Diderot measured radioactivity in Paris' 18th arrondissement and held an Open Day at the institute to explain their measurements to local residents. During an emergency response exercise organised in the vicinity of Golfech power plant, pupils at Valence secondary school in Agen measured ambient radioactivity, as they might have to do in the event of a real emergency, and logged their results on the OpenRadiation and Safecast websites.

Educational initiatives using OpenRadiation have also been developed, based on special events, such as accompanying Year 10 pupils from schools in the Essonne to Fête de la Science, France's national science festival, to set up projects, or organising OpenRadiation dosimeter kit assembly workshops.

\section{Conclusion}

From this early feedback the OpenRadiation project, we can see that the public has a keen interest in using this kind of tool to take measurements and share the results in real time. Feedback from certain users has already been taken into account to make the app more user-friendly and this will continue in the future, in particular with a view to upgrading the website and its functions.

In general, the measurements collected are of good metrological quality, which shows that users have understood the tool and the related good practices. This quality will be a determining factor in the future possibilities and outlook for using the measurements, which are likely to be extremely diverse, and for which practical applications are already being implemented.

The first two years since the OpenRadiation project was launched have shown that the public is increasingly interested in the project and user communities are emerging, especially in secondary schools and in the context of research projects. More projects are being developed, involving new user communities, like the local information committees that have been formed near nuclear facilities in France. The project's partners, and its governance, will have to change in line with the development of user communities, and to deal with an increasingly international dimension.

\section{References}

Bertho JM, Maître M, Crouail P, Naito W, Shkliarava N, Mostovenko A, Jones K, Simon-Cornu M. 2019. Assessment of population radiation exposure at the edge of the exclusion zone 32 years after the Chernobyl accident: Methods and preliminary results. Radioprotection 54(4): 247-257

Bottollier-Depois JF, Blanchard P, Clairand I, Dessarps P, Fuller N, Lantos P, Saint-Lô D, Trompier F. 2007. An operational approach 
for aircraft crew dosimetry: The SIEVERT system. Radiat. Prot. Dosim. 125(1-4): 421-424.

Brown A, Franken P, Bonner S, Dolezal N, Moross J. 2016. Safecast: Successful citizen-science for radiation measurement and communication after Fukushima. J. Radiol. Prot. 36(2): S82.

IRSN. 2016. Exposition de la population française aux rayonnements ionisants. ed IRSN, p. 23 (in French).

Lochard J, Schneider T, Ando R, Niwa O, Clement C, Lecomte JF, Tada JI. 2019. An overview of the dialogue meetings initiated by
ICRP in Japan after the Fukushima accident. Radioprotection 54 (2): $87-101$

Schneider T, Maître M, Lochard J, Charron S, Lecomte JF, Ando R, Kanai Y, Kurihara M, Kuroda Y, Miyazaki M, Naito W, Orita M, Takamura N, Tanigawa K, Tsubokura M, Yasutaka T. 2019. The role of radiological protection experts in stakeholder involvement in the recovery phase of post nuclear accident situations: Some lessons from the Fukushima-Daïchi NPP accident. Radioprotection 54(4): 259-270

Cite this article as: Bottollier-Depois JF, Allain E, Baumont G, Berthelot N, Darley G, Ecrabet F, Jolivet T, Lebeau-Livé A, Lejeune V, Quéinnec F, Simon C, Trompier F. 2019. The OpenRadiation project: monitoring radioactivity in the environment by and for the citizens. Radioprotection 54(4): 241-246 